Cinémas

Revue d'études cinématographiques

Journal of Film Studies

\title{
L'historiographie parallaxiale et la flâneuse : le cinéma pré- et postclassique
}

\section{Catherine Russell}

Volume 10, numéro 2-3, printemps 2000

Cinéma et intermédialité

URI : https://id.erudit.org/iderudit/024820ar

DOI : https://doi.org/10.7202/024820ar

Aller au sommaire du numéro

Éditeur(s)

Cinémas

ISSN

1181-6945 (imprimé)

1705-6500 (numérique)

Découvrir la revue

Citer cet article

Russell, C. (2000). L'historiographie parallaxiale et la flâneuse : le cinéma préet postclassique. Cinémas, 10(2-3), 151-168. https://doi.org/10.7202/024820ar

\section{Résumé de l'article}

L'historiographie parallaxiale renvoie à la manière par laquelle le cinéma des premiers temps apparaît lorsqu'il est considéré selon la perspective de la fin du XXe siècle. Le présent article traite des parallèles qu'on peut établir entre la première décennie du cinéma et la diversification des médias visuels dans les deux dernières décennies du siècle. Ces similitudes ont été théorisées par les chercheuses féministes Miriam Hansen, Anne Friedberg et Giuliana Bruno, qui ont soutenu que les sphères publiques caractéristiques de ces deux périodes ont engendré une forme de spectatorat très différente de celle que proposait la " théorie du dispositif ». La diversité des médias et des positions de lecture, ainsi que les diverses architectures de réception, semblent renvoyer à un certain modèle de modernité et à un regard qui offre un espace au spectatorat féminin. Le présent article tente de résumer et de rassembler ces théories en insistant particulièrement sur leur valeur à titre d'historiographie du cinéma des premiers temps. 


\section{L'historiographie parallaxiale et la flâneuse : le cinéma pré- et postclassique ${ }^{1}$}

\section{Catherine Russell}

\section{RÉSUMÉ}

L'historiographie parallaxiale renvoie à la manière par laquelle le cinéma des premiers temps apparaît lorsqu'il est considéré selon la perspective de la fin du XXe siècle. Le présent article traite des parallèles qu'on peut établir entre la première décennie du cinéma et la diversification des médias visuels dans les deux dernières décennies du siècle. Ces similitudes ont été théorisées par les chercheuses féministes Miriam Hansen, Anne Friedberg et Giuliana Bruno, qui ont soutenu que les sphères publiques caractéristiques de ces deux périodes ont engendré une forme de spectatorat très différente de celle que proposait la "théorie du dispositif». La diversité des rnédias et des positions de lecture, ainsi que les diverses architectures de réception, semblent renvoyer à un certain modèle de modernité et à un regard qui offre un espace au spectatorat féminin. Le présent article tente de résumer et de rassembler ces théories en insistant particulièrement sur leur valeur à titre d'historiographie du cinéma des premiers temps.

\section{ABSTRACT}

Parallax historiography refers to the way that early cinema comes into focus from the perspective of the end of the 20th century. This essay examines the parallels berween the first decade of cinema and the diversification of visual media in the $1980 \mathrm{~s}$ and $90 \mathrm{~s}$, similarities which have been theorized by the feminist film scholars Miriam Hansen, Anne Friedberg and Giuliana Bruno. 
These theorists have argued that the public spheres of these two periods solicit a very different form of spectatorship than that theorized by "apparatus theory". The diversity of media and of viewing positions, as well as the various architectures of reception, suggest a model of modernity and a gaze that offers a space for female spectatorship. This essay summarizes and collates these theories, foregrounding their value as a historiography of early cinema.

Au début des années quatre-vingt-dix furent publiés trois livres qui, considérés conjointement, jettent un éclairage neuf sur la première décennie de l'histoire du cinéma. Babel and Babylon de Miriam Hansen, Window Shopping d'Anne Friedberg et Streetwalking on a Ruined Map de Giuliana Bruno constituent une historiographie fondée pour l'essentiel sur la transformation de la culture visuelle à la fin du XX siècle. Bien que l'entreprise n'ait pas été concertée, elle témoigne de démarches similaires et d'une préoccupation commune. Établissant des parallèles entre les débuts du cinéma et le cinéma postmoderne, les auteures élaborent une théorie du spectatorat qui met en question la position unitaire et transcendante du spectateur de la période classique: dans le cinéma pré- et postclassique, le spectatorat apparaît instable et hétérogène, fluide et mobile; sa posture n'est plus celle de la "maîtrise», dont on sait qu'elle a été théorisée depuis une vision masculine et bourgeoise. Quant au "cinéma classique ", il apparaît comme une période intermédiaire de l'histoire du cinéma.

Mon propos ici est d'explorer les parallèles entre les cultures visuelles du début et de la fin du $\mathrm{XX}^{\mathrm{e}}$ siècle et de mesurer les effets historiques de cette historiographie "parallaxiale ", l'épithète évoquant à la fois le changement de perspective et la notion de parallélisme. Que nous dit cette historiographie, tant sur l'histoire que sur le cinéma? Je soutiendrai qu'il s'agit là d'une forme d'historiographie féministe peu reconnue et sous-estimée, qui en fait constitue un véritable défi à l'hégémonie du classicisme et à ses prolongements dans le discours des études cinématographiques.

Il est vrai que plusieurs des observations faites par l'historiographie parallaxiale sont analogues aux positions d'autres histo- 
riens des débuts du cinéma. Les trois auteures reprennent notamment la notion de "cinéma des attractions» que Tom Gunning a proposée pour souligner la façon dont les films d'avant 1907 s'adressaient directement au spectateur en "sollicitant son attention et sa curiosité par des actes d'exhibition" (1994, p. 190). Plutôt que de privilégier les fonctions narratives, le cinéma des attractions met l'accent sur ses liens avec d'autres formes contemporaines de divertissement (le cirque, le théâtre de vaudeville et le peepshow); plutôt que d'absorber le spectateur, ce cinéma "reconnaît son propre processus de déploiement " (p. 191). En développant sa réflexion sur le cinéma des premiers temps (1991 et 1989), Gunning a établi certains parallèles avec l'avant-garde. Et c'est en partie grâce à ces rapprochements entre le cinéma d'avant-garde des années 1960-1970 et le cinéma du début du siècle si, à la fin des années 70 , les chercheurs ont tenté de repenser le cinéma préclassique en tant que pratique intégrée et autonome qui, à ce titre, méritait un examen plus approfondi. Cette mise entre parenthèses de la période classique, la réduction du cinéma classique à un simple intermède au milieu du XXe siècle, constitue assurément une conséquence majeure de ce nouveau type d'historiographie. Miriam Hansen elle-même a posé une série de questions à propos des parallèles qui existent entre le cinéma des premiers temps et ce qu'elle appelle le cinéma "récent": "Quel est le but d'une telle comparaison? Comment la rendre productive, au-delà d'une simple analogie formaliste, au-delà de la nostalgie ou d'un pessimisme culturel ? Comment juxtaposer ces deux périodes sans supprimer leurs différences historiques (1994, p. 140)?

La réponse de: Miriam Hansen à ces questions consiste à privilégier des processus de transformation plus larges dans le développement de la sphère publique au début et à la fin du $\mathrm{XX}^{\mathrm{e}}$ siècle. Selon elle, la possibilité pour le cinéma de produire une "sphère publique alternative" est manifeste dans les formes de réception et d'exposition propres à ces deux périodes. Autrement dit, pour cette historiographie parallaxiale, il n'y pas de véritable analogie formelle entre l'avant-garde et le «cinéma primitif ". Hansen se concentre plutôt sur le caractère interactif de la relation film-public dans ces périodes, ainsi que sur les 
différentes formes à travers lesquelles ces «territoires divers et marginalisés " sont appréhendés. Le cinéma du début et le cinéma récent, soutient-elle, n'ont pas un effet homogénéisant sur la diversité des spectateurs, mais favorisent au contraire la formation de sous-cultures de réception. L'intermède historique constitué par le cinéma classique souligne également la brièveté de la "culture de masse", qui a peut-être dominé la culture visuelle des années 1920-1960 (1994, p. 136) mais qui, d'autre part, a ouvert la porte à la "diversification des médias électroniques globaux » à laquelle on assiste aujourd'hui (p.148).

Le modèle de spectatorat que Bruno, Friedberg et Hansen décrivent doit être vu comme une théorie alternative à celle du dispositif. Par l'inscription du cinéma des premiers temps dans un espace culturel complexe qui inclut l'architecture, le théâtre, le journalisme et le divertissement populaire, l'acte de regarder un film est perçu comme une activité quotidienne. De plus, la mobilité du spectateur placé au carrefour de ces diverses formes de spectacle et le rôle de premier plan que joue l'intertextualité dans le cinéma de cette période donnent un caractère fortement interactif à l'activité du spectateur. C'est à travers ce rôle complémentaire de l'intermédialité et de l'interactivité que le parallèle entre le cinéma des débuts et le cinéma postmoderne commence à faire sens. Si, dans la théorie du dispositif, la position spectatorielle est associée à des formes transcendantes de conscience ou à l'illusion d'une maîtrise visuelle, le spectateur du cinéma des premiers temps et du cinéma récent apparaît en contrepartie comme une construction incarnée, socialement configurée et hétérogène. Le cinéma classique se trouve dès lors associé à la théorie du dispositif et désigne une période où le cinéma acquiert une certaine autonomie par rapport aux autres formes médiatiques de représentation.

Bien que l'historiographie parallaxiale se soit développée en même temps que se produisaient des changements réels dans la production, l'exploitation et la réception cinématographiques, elle implique cependant une critique de l'association entre le cinéma classique et la théorie du dispositif. Il faut se méfier de la confusion entre une période historique et une théorie du spectatorat. En fait, aucune des auteures ne va jusqu'à faire une telle 
critique, quoique le "cinéma récent" soit défini par Hansen et Bruno comme une rupture d'avec le classicisme. En défendant et en élaborant une théorie alternative du spectatorat, ces théoriciennes touchent aux fondements mêmes de la période classique. Dans sa discussion sur Rudolph Valentino et le spectatorat féminin dans les années vingt, Hansen décrit une spectatrice qui lit le texte classique "contre le grain" et encourage un point de vue qui tient compte d'une diversité à l'intérieur du "cinéma classique" (1991, p. 245-268). L'effet le plus radical de l'historiographie parallaxiale pourrait bien être une ultime remise en question de l'existence du cinéma classique et de sa forme constitutive de spectatorat. Le cinéma classique n'était peut-être, après tout, qu'une manière spécifique de voir et de théoriser le film. L'appel de Laura Mulvey à un "détachement passionné " a peut-être toujours été une possibilité pour le spectateur, mais il ne s'est complètement réalisé que dans le cinéma des débuts et le cinéma récent, précisément en raison de leurs structures de réception.

En constatant la dispersion et la fragmentation des modes de spectatorat communs au cinéma des premiers temps et au cinéma récent, nous pouvons peut-être mieux comprendre l'importance de l'architecture dans le "positionnement» du spectateur. Lidéal du cinéma narratif classique, théorisé par Christian Metz, est d'aligner le regard du spectateur sur la ligne de vision de la caméra ou du projecteur. On comprend rétrospectivement que cela constitue une dénégation de la vision qui regarde «de près ", du coup d'œil à côté, et de la possibilité même d'assumer de telles positions. Une telle conception du classicisme comme dispositif monolithique tend à annuler certaines distinctions importantes entre les genres et les modes de pratique filmique, qui sollicitent en fait des formes très différentes de spectatorat (Williams). Pour l'historiographie parallaxiale, la théorie du dispositif est une production idéologique de la culture de masse, une construction qui reproduit dans la théorie une architecture particulière de réception.

L'importance de l'architecture en tant que contexte de vision des films est l'une des principales contributions du travail de Giuliana Bruno sur le cinéma des premiers temps. Son livre est 
consacré principalement aux films d'Elvira Notari, une cinéaste napolitaine prolifique dont le travail a été plus que négligé par les historiens du cinéma italien. Les films de Notari, dont plusieurs furent tournés dans les rues de Naples, étaient vus dans des salles intégrées à ces immenses Gallerias, sortes de centres commerciaux vitrés qui constituaient des carrefours d'activités sociales à la fin du XIX ${ }^{e}$ siècle en Europe. Bruno décrit ici la version napolitaine d'un phénomène qui existait dans la plupart des centres métropolitains:

La Galleria a prolongé les fonctions de la piazza (forum), site urbain en Italie qui est un lieu de rencontres et de promenades, d'événements sociaux et d'activités de passage. Ce sire représentait la coalescence et la transformation de la vie publique, traditionnellement organisée autour de la piazza urbaine, dans un contexte moderne. Logé dans les arcades, le cinéma se trouvait ainsi au cœur d'un lieu de spectacles, de circulation de personnes et de marchandises, un site métropolitain très diversifié socialement, fréquenté autant par les élites et l'intelligentsia que par les classes défavorisées et les groupes marginaux (p. 43).

Ces Arcades sont au cour d'un long travail inachevé dans le domaine des études culturelles, celui de Walter Benjamin. Il est important de se rappeler que Benjamin considérait ces structures comme les ruines allégoriques du XIX siècle. Il les concevait comme des "passages" permettant de traverser le temps et l'espace. Bruno et Friedberg ont pris cette idée de Benjamin comme point de départ de leurs propres travaux sur le cinéma des premiers temps, considérant ainsi leur objet d'étude dans une perspective qui ne serait développée que beaucoup plus tard. Logé dans ces passages ou Arcades, le cinéma est vu comme l'emblème d'une version de la modernité qui remet en question celle du cinéma classique. Il s'agit en quelque sorte d'une modernité inversée, qui regarde "en arrière vers le futur" (back to the future) dans le but de cerner la formation d'un sujet moderne différent. Les trois auteures étudiées dans le présent article citent le travail le plus célèbre de Benjamin, "L'œuvre d'art à l'époque de sa reproduction mécanique" : 
Nos bistros et nos avenues métropolitaines, nos bureaux et pièces meublés, nos gares et nos usines paraissaient devoir nous enfermer sans espoir de jamais y échapper. Puis vint le film, qui fit sauter ce mondeprison, déchiré en un dixième de seconde comme par une dynamite, si bien que désormais, au milieu de ces débris projetés au loin, nous partons calmement et aventureusement en voyage (1969, p. 236).

On sait que Benjamin ne s'intéressait pas seulement à l'implantation du cinéma dans les Arcades au tournant du siècle, mais aussi à son rôle dans la culture urbaine et métropolitaine. Il associait les effets cinématographiques de montage et de construction spatiale à l'expérience psychologique qui résulte de la vie dans une grande ville. Les stimuli et les chocs provoqués par le trafic, la foule, la densité et la fragmentation de l'espace, sont pour lui des facteurs déterminants des deux premières décennies de la culture cinématographique (Singer, 1995). La notion de "choc" proposée par Benjamin était une façon de décrire à la fois l'esthétique des débuts du cinéma et l'expérience quotidienne du spectateur, dans laquelle le cinéma était toujours présent de manière implicite.

Les théories de Benjamin sur le cinéma et la modernité sont instructives à maints égards pour l'historiographie parallaxiale du cinéma des premiers temps et du cinéma récent. La "perte de l'aura " qu'il avait attribuée à la reproduction mécanique de l'art est à la base de ce genre d'historiographie, dans la mesure où les médias électroniques représentent une autre étape du processus de transformation de la sphère publique. La perte de l'aura ne manifeste pas une attitude nostalgique pour ce qui disparaît; elle s'en prend plutôt à une conception utopique des transformations historiques. S'opposant à une historiographie téléologique ou évolutionniste $\mathrm{du}$ "progrès", Benjamin plaide en faveur d'une historiographie dialectique. Dans le contexte de son projet sur les Arcades, il écrit:

L'image dialectique est un éclair. L'Autrefois doit être capturé rapidement lorsqu'il fait briller son imageéclair dans le Maintenant du reconnaissable. Ainsi dans le moment, et seulement ainsi, nous pouvons sauver ce 
qui, le moment suivant, est déjà irrémédiablement perdu (1983a, p. 64).

La correspondance entre le cinéma des premiers temps et le cinéma récent peut être conçue comme une relation dialectique dans laquelle l'ère électronique occupe le même rôle que celui que jouèrent les techniques photographiques dans les premières décennies $\mathrm{du} \mathrm{XX}^{\mathrm{e}}$ siècle. La vidéo et les techniques numériques de l'image menacent ainsi de supplanter la "sphère publique" du cinéma classique et les formes constitutives de sa subjectivité. Si Benjamin reconnaît dans le cinéma les traces de formes d'expériences antérieures qui se prolongent dans ce qu'il appelle allégoriquement "le pays de la technologie", on peut se demander s'il existe un effet rédempteur analogue dans cette seconde étape, celle du passage aux nouvelles techniques de représentation à la fin du XXe siècle.

Giuliana Bruno prend l'exemple du cinéma présenté dans les avions, qu'elle considère comme une incarnation récente de l'implantation d'un média dans une architecture de transition. Elle prétend qu'en "incarnant les dynamiques du voyage, le cinéma crée une topographie hétérotopique", "un site dont le système d'ouverture et de fermeture à la fois l'isole et le rend impénétrable, créant une sorte d'ailleurs/nulle part" (p. 57). Voilà une description qui pourrait correspondre aussi bien à Internet, quoiqu'elle se rapporte au cinéma des premières décennies du $\mathrm{XX}^{e}$ siècle. Dans un article plus récent, Bruno a développé ses observations sur les architectures du cinéma des premiers temps en une théorisation plus large de l'expérience cinématographique, qu'elle décrit comme une habitation tactile et transitoire de l'espace :

Enfermé dans un regard figé, le spectateur était transformé en voyeur. Parler de site de vision [siteseeing] implique que, en raison de la cinétique spatio-temporelle du film, le spectateur est un voyageur plutôt qu'un voyeur. Par ce passage à un voy(ag)eur, mon objectif est de revendiquer une mobilité féminine, et, prenant la position d'une voyageuse (de films), de défendre l'idée que le film est une cartographie moderne. Il constitue une carte mobile (1997, p. 10). 
La relation d'opposition qui s'établit entre l'implantation du cinéma muet dans la métropole et le voyage permis par Internet et par le zapping télévisuel est implicite dans la réflexion de Bruno sur le spectatorat. Le renversement d'un certain régime de la culture visuelle pour lequel l'œil est un instrument clé constitue pour elle le défi féministe aux théories classiques du cinéma, dominées par les notions de voyeurisme et de mâ̂trise.

Hansen prétend pour sa part que c'est le marché de la vidéo qui a fait «[...] du spectateur classique l'objet d'une contemplation nostalgique" (1991, p. 3). C'est dans ce contexte d'une aura en désintégration que la sphère publique alternative, "[...] en tant qu'horizon d'expérience hétérogène et, parfois, imprévisible», peut se concrétiser. La pluralité de ce spectatorat est inscrite à l'intérieur des lieux et des conditions de réception - en plus des différents modes de communication textuelle, que le cinéma classique a cherché à standardiser et à universaliser. C'est pourquoi l'historiographie parallaxiale va même jusqu'à considérer la pratique visuelle contemporaine comme le développement de nouvelles formes de spectatorat. Des formes qui peuvent avoir certaines similitudes avec la culture visuelle d'il y a cent ans, mais qui, de manière significative, sont en train de transformer l'expérience de la sphère publique du cinéma classique, pour le meilleur ou pour le pire, en une forme d'expérience en voie de disparition.

Friedberg va plus loin que Bruno et Hansen dans sa description des changements de la culture visuelle provoqués par les nouveaux médias électroniques. Elle croit que le spectatorat télévisuel constitue une subjectivité particulière, caractérisée surtout par ses dislocations temporelles. Pour Friedberg, le bouleversement significatif de la sphère publique, conséquence de l'arrivée des médias électroniques, est relié aux possibilités qu'offre le magnétoscope maison (l'enregistrement d'émissions diffusées par plusieurs chaînes, la capacité de manipuler le temps narratif lorsqu'on recule ou avance la bande vidéo, etc.). De plus, elle associe cette mobilité temporelle à la mobilité spatiale caractéristique des multiplexes dans les centres commerciaux: le spectateur doit chercher les films, que ce soit chez les marchands de vidéos, dans les centres commerciaux, à travers les chaînes télévisuelles 
spécialisées, ou encore, en naviguant sur Internet (quoique Friedberg n'inclue pas ce dernier cas dans son livre, qui date de 1993).

Friedberg est aussi celle qui trace le plus minutieusement les parallèles entre les débuts du cinéma et le cinéma postmoderne. Son modèle du «regard virtuel mobile" permet de lier les deux périodes, et aussi de les distinguer du cinéma classique. Avant d'être inscrite dans la représentation cinématographique, la mobilité spatio-temporelle du regard s'est développée au XIX ${ }^{e}$ siècle à travers les différentes formes de tourisme et de voyages à l'étranger. Quant à l'aspect virtuel du regard, il précède le cinéma à travers les dioramas, les panoramas, les musées de cire et la photographie. Le regard mobile et virtuel se rapporte au consommateur-sujet (consumer-as-subject) dans la culture visuelle. Friedberg prétend que ce spectateur-acheteur (spectatorshopper) "[...] essaie d'enfiler différentes identités" et voyage dans le temps à travers les films et l'histoire culturelle. Si les discussions en direct par l'intermédiaire d'Internet (chat rooms) représentent l'apothéose de cet "essai de différentes identités", le cinéma classique en contrepartie apparaît comme un retour à une forme plus ancienne caractéristique du XIX siècle, une sorte de piège discursif qui empêchait le média de s'accomplir véritablement en tant que phénomène émanant d'une culture urbaine et globale.

Friedberg soutient que la postmodernité «[...] est marquée par la centralisation grandissante des caractéristiques qui sont implicites à l'acte de regarder un film". Elle poursuit en affirmant que "[...] la subjectivité de la "condition postmoderne" est le résultat d'une accélération industrielle des fluidités spatiales et temporelles» —- dynamique qui fut commercialisée au milieu du XIX siècle (p. 179). Cette théorie implique ainsi que la technologie électronique de l'image est la réalisation des divers effets de la modernité originairement inscrits dans le cinéma, mais reconnaissables seulement à la fin du $X X X^{e}$ siècle. Vu sous cet angle, le cinéma apparaît comme une sorte de passage à travers le temps qui nous permet de saisir différemment la modernité. L'historiographie parallaxiale du cinéma des premiers temps et du cinéma récent ne concerne donc pas vraiment le cinéma, mais bien la modernité; les différentes approches formalistes et 
narratologiques des études cinématographiques n'ont fait que masquer cette modernité de l'espace social que le cinéma continue de construire et de reconstruire.

$\mathrm{Si}$ les nouveaux médias électroniques et les formes numériques de représentation constituent une certaine perte de l'aura cinématographique, c'est toutefois la référentialité elle-même qui se perd. La "secondarité" de l'allégorie benjaminienne est devenue, en cette ère du traitement numérique de l'image, une forme plus radicale de perte de signification. Notons toutefois que la théorie de la représentation de Benjamin est déjà construite sur une temporalité du déplacement et de la perte, et si l'historiographie parallaxiale nous encourage à percevoir et à saisir différemment la culture de l'image, je pense qu'elle met au premier plan la fonction d'archivage des pratiques postmodernes de recyclage. Par exemple, il est possible aujourd'hui, sur le site Internet de l'American Mutoscope and Bioscope Company, de visiter ses studios new-yorkais des années 1890 et d'acheter toutes sortes de choses (des cassettes vidéo aussi bien que des écrans de sauvegarde pour l'écran d'ordinateur). Cette mise en marché du cinéma des premiers temps à la fin des années 1990 n'est pas seulement rendue possible par Internet: ce sont les nouveaux médias qui ont fait de cette période du cinéma un produit consommable. Ce qui se perd en fait d' "expérience de l'aura» est redonné par l'accroissement du répertoire de la culture visuelle, un répertoire dans lequel la perte même de cette expérience se trouve inscrite sans ambiguité.

Les techniques numériques de l'image permettent aussi de réhabiliter la culture des médias par l'intermédiaire de leur fonction d'archivage. Pour ne citer qu'un exemple, la compagnie Kino Video a récemment offert aux consommateurs d'images une compilation de films des frères Lumière en DVD, chose inconcevable il y a à peine quelques décennies. Dans la section consacrée aux voyages, nous nous déplaçons de Venise à Londres, puis vers Dublin jusqu'à Belfast et Berlin. Les scènes situées à Londres et à Berlin montrent des salles mettant en vedette le cinématographe. Nous pouvons dès lors regarder les films des frères Lumière, non pas simplement en tant qu'images de villes, mais bien en tant que manières de regarder ces villes, 
cas exemplaire de ce que Friedberg décrit comme le «regard virtuel mobile", qui est discontinu tant d'un point de vue spatial que temporel. Je crois que cette forme de spectatorat est un élément clé de la notion de "cinéma récent" développée par l'historiographie parallaxiale. Lorsque nous louons des films sur vidéocassettes, nous consommons des moments d'histoire; et ce ne sont pas uniquement les professeurs et les étudiants en cinéma qui le font.

Cette version de la modernité élaborée par l'historiographie parallaxiale trouve son modèle le plus soutenu dans le concept du flâneur, présenté par Charles Baudelaire comme le «spectateur passionné" de "la grâce scintillante de tous les éléments de la vie» (p. 9). L'homme de la foule qui transforme l'espace urbain en un kaléidoscope d'images, qui est à la fois détaché et partie intégrante du spectacle, devient pour Benjamin la figure de l'héroïsme de la vie moderne, figure essentiellement mélancolique (1983b, p. 66). La flânerie représente l'activité même de vivre en public. Pour le flâneur nomade, subjectivité en transit, la rue devient une demeure, les édifices et la configuration géographique de la ville constituent les murs des pièces dans lesquelles il habite. Le cinéma, en tant que prolongement de la flânerie, va inverser cette mobilité spatiale, faisant de l'intérieur du théâtre un espace extérieur dans lequel il peut vagabonder. La flânerie se rapporte également à une temporalité proprement moderne. Si le flâneur de Benjamin voit dans une culture de la consommation les traces de l'expérience du passé, le spectateur d'un film voit de la même façon la "réalité" comme une représentation allégorique et secondaire - comme une virtualité. La figure du flâneur réunit ainsi les thèmes clés de l'historiographie parallaxiale: une dislocation spatiale et temporelle et un spectatorat mobile qui s'inscrit dans une sphère publique de la culture visuelle, où le cinéma fait partie d'un champ plus large de technologies, de médias et d'architectures de réception.

Pour Baudelaire et Benjamin, le flâneur est indiscutablement masculin. Parmi les attractions de choix que lui offrait la ville, nous retrouvons la prostituée, figure qui elle aussi parcourt les rues de la ville, comme nous le rappelle le titre du livre de Bruno. Dans le Paris du milieu du XIX ${ }^{c}$ siècle, époque où ce discours mo- 
derniste se développe, les femmes faisaient partie intégrante de la fantasmagorie de cette culture de consommation, évoquant chez le poète mâle un désir complexe pour un objet fabriqué en série, un objet moderne. Elles apparaissent comme des emblèmes du caractère intersubjectif de la flânerie, des objets qui "détournent le regard" d'une manière distanciée et allégorique. Un des éléments qui attire tout particulièrement Benjamin dans la poésie de Baudelaire est le regard figé des femmes, dont les yeux sont "lustrés", "illuminés comme les vitrines de boutiques" (1983b, p. 150). Ce regard figé et mystérieux de la femme est très présent dans la poésie de Baudelaire, mais c'est un regard dangereux et furtif qui ne peut éliminer l'anxiété du flâneur. La femme de la rue provoque donc une forme particulière de subjectivité moderne, mais est elle-même privée de subjectivité.

Le féminisme contemporain s'est débattu avec la notion de flâneuse, l'incarnation féminine du flâneur. Janet Wolff maintient que ce concept est impossible en raison d'une construction sexualisée de la modernité (engendering of modernity) qui ne tient pas compte de la présence des femmes dans la sphère publique (p. 47). Il était impossible pour les femmes se promenant "dans la rue» d'atteindre le niveau d'invisibilité du flâneur alors que les hommes pouvaient de facto observer la parade de la modernité sous le couvert de l'anonymat, les femmes avaient besoin de se travestir afin d'en arriver à ce genre de liberté. Ainsi, George Sand devait emprunter les habits et les manières du flâneur afin de circuler librement dans Paris (Wolff, p. 148; Gelber, p. 59). Cependant, Friedberg et Bruno ont toutes deux révisé cette notion de flâneuse dans l'intention de retrouver le pouvoir du regard féminin, qui reste latent dans la description qu'en fait Baudelaire. Alors que ce regard n'était pour Baudelaire qu'un simple "coup d'œil " (glance), il a été en fait développé comme un "regard attentif» (gaze) par la culture de consommation émergente. Friedberg prétend que la consommatrice du $\mathrm{XIX}^{e}$ siècle était directement ciblée en tant que flâneuse dans les magasins à rayons et dans les arcades. De plus, l'accent de plus en plus important que l'on mettait sur les vitrines des boutiques, établissant ainsi une relation entre l'acte de regarder et l'acte d'acheter, construisait la flâneuse en tant que spectatrice mobile. 
Le regard virtuel et mobile institutionnalisé dans le cinéma trouve donc ses origines dans la culture de consommation et dans ses formes coextensives de subjectivité féminine. Bruno va approfondir cette version de la flâneuse en l'inscrivant dans l'espace psychologique de la fantaisie ouvert par le cinéma:

Le cinéma donnait aux femmes une forme d'accès à l'espace public, une occasion de socialiser et de sortir de la maison. Aller au cinéma déclenchait une sorte de libération du regard de la femme, qui lui permettait de renégocier la configuration privé/public dans un nouvel espace d'intersubjectivité (1993, p. 51).

Le spectatorat féminin, dans ce cadre théorique du modèle de la flâneuse, apparaît comme une fonction du "magasinage" et de la consommation. Dans la mesure où le cinéma est inscrit dans la culture de consommation, il permet de concrétiser la possibilité pour la culture visuelle d'offrir aux femmes une sphère publique alternative. C'est un modèle qui tend à nier les formes narratives de la formation de l'identité et du sujet, au profit d'une conception plus large de l'acte d'aller au cinéma, qui apparaît comme un des nombreux plaisirs qu'offre la ville. Miriam Hansen n'évoque pas comme telle la figure de la flâneuse, mais elle décrit cependant la sphère publique alternative du cinéma des premiers temps comme un espace où " [...] les conditions de possibilité [d'] une tradition de spectatorat féminin" peuvent être retracées (1991, p 125). Elle admet volontiers que cette tradition ne peut être mesurée «au sens empirique du terme", mais que des indices textuels et extratextuels suggèrent qu'il y avait, dans la sphère publique du cinéma muet, "une menace soutenue commercialement contre le monopole du regard masculin" (p. 121).

Le cinéma des premiers temps se trouve donc associé à la consommation et aux autres formes de divertissement de la même façon que, à la fin du $\mathrm{XX}^{\mathrm{e}}$ siècle, les théâtres contemporains et la publicité télévisée mettent en contexte la manière de regarder les films. On pourrait également donner l'exemple du zapping télévisuel ou de la navigation dans Internet, qui apparaissent aussi comme des modes parallèles de spectatorat. Pourtant, ces modes 
ne nous semblent pas particulièrement ou nécessairement liés à la spectatrice. Et ce n'est peut-être qu'à travers l'effet parallaxial de ce type d'historiographie qu'il pourront à leur tour devenir le terrain de la flâneuse. En fait, les parallèles entre les formes contemporaines de spectatorat et celles du cinéma des premiers temps ne sont que partiels, car dans la mesure où nous sommes témoins de la prolifération de nouveaux modes de consommation des films à la maison, sorte de domestication de la culture visuelle, nous assistons à une régression du cadre urbain du cinéma. La sphère publique du cinéma est aujourd'hui reconfigurée en tant que "géographie d'urgence" impliquant des moments, des lieux et des spectateurs disparates (Corrigan). Plusieurs théoriciens ont déjà décrit la temporalité propre à la culture vidéo comme non linéaire et instantanée - une sorte d'état perpétuel de catastrophe, dans lequel l'effet de fragmentation de la culture visuelle a atteint un niveau fondamentalement différent de celui de la culture métropolitaine du début du $\mathrm{XX}^{\mathrm{e}}$ siècle (Corrigan et Doane).

Si les villes accumulent en couches stratifiées les traces et les ruines des générations précédentes, les techniques numériques produisent de nouvelles structures de commémoration : les fragments sont réarrangés et recontextualisés dans de nouvelles architectures de réception tels la salle de classe, le film de compilation et les banques d'images sur support électronique. Ainsi réinscrit dans les archives, le cinéma des premiers temps est repensé et reconstruit en suivant les traces de l'invisible et impossible flâneuse. La modernité fait l'objet d'une dynamique sexuelle différente, alors que les cultures de consommation de l'image entrent en collision. Il s'agit d'une forme d'historiographie inventée et fantastique, presque littéraire, fondée beaucoup plus sur le désir contemporain de trouver de nouveaux modèles de spectatorat féminin que sur des preuves empiriques ou de la documentation historique. Pourtant le regard virtuel mobile est aussi un regard dans l'histoire, un regard qui aborde le cinéma comme une "machine à voyager dans le temps", machine qui peut toujours nous ramener non seulement vers les lieux nostalgiques de la mémoire linéaire, mais également vers les chocs non linéaires provoqués par un retour vers le futur. 
Le reconnaissance du passé en tant que phénomène intelligible pour nous aujourd'hui, dans la configuration benjaminienne de l'image-éclair ou de l'«éveil» de l'image-dialectique, est une forme d'historiographie différente de celle qui est proposée par un auteur comme Jean Baudrillard, qui affirme qu'après la linéarité du "progrès" moderne, quelque part dans les années 1980, l'histoire a pris une nouvelle direction: "[...] dans notre espace non euclidien, en cette fin de siècle, une courbure malveillante détourne invinciblement toutes les trajectoires... Chaque mouvement apparent de l'histoire nous rapproche imperceptiblement de son antipode, voire de son point de départ" (1999). Les nombreuses festivités mondiales entourant le centenaire du cinéma semblent en effet corroborer ce que Baudrillard a décrit comme "un travail de deuil mal dirigé ou raté, qui cherche à tout réviser, réécrire, restaurer et rajeunir». Il se demande «[...] si le mouvement de la modernité est réversible, et si, en retour, cette réversibilité serait irréversible. Jusqu'où peut aller cette activité rétrospective, ce rêve de fin de millénaire?"

Je répondrai à cela que l'historiographie parallaxiale du cinéma des premiers temps et du cinéma récent n'implique pas simplement une réécriture ou une réversibilité de l'histoire. Elle conçoit plutôt cette dernière comme un panorama qui change en fonction du regard de l'observateur. D'autres regards, parallèlement à ceux de la flâneuse, ont ainsi été jetés sur les débuts du cinéma. Par exemple, Fatimah Tobing Rony a décelé ce qu'elle nomme un «troisième cil" dans les premières représentations ethnographiques expérimentales. Si Baudrillard est à la recherche d'une "orbite temporelle alternative" qui permettrait "de prendre un raccourci elliptique, et de passer au-delà de la fin, en ne lui laissant pas le temps d'avoir lieu ", il faut souligner ici que c'est précisément l'effet recherché par l'historiographie parallaxiale. En fait, les nouveaux modes de spectatorat qui ont été "découverts" dans le cinéma des premiers temps n'ont pas été expérimentés comme tels par les gens de l'époque. La flâneuse n'a pas existé, car elle ne pouvait pas être reconnue dans la construction effectuée par le regard moderne. Maintenant que nous savons qui elle est, nous pourrons tenter de la repérer dans les nouveaux médias du XXl siècle. L'aura du cinéma devient ainsi 
liée à celle d'une modernité en train de disparaître avec les techniques analogiques qui maintenaient en place une forme particulière de spectatorat. Aussi l'historiographie parallaxiale du cinéma des premiers temps et du cinéma récent apparaît-elle comme une projection féministe fondamentalement utopique se situant dans le cadre de la sphère publique postmoderne.

Le cinéma n'est peut-être pas "mort", mais au fur et à mesure qu'il s'entremêle à la vidéo et aux techniques numériques, son autonomie pourrait bien être remise en question. L'historiographie parallaxiale du cinéma nous encourage à repenser l'histoire du cinéma à la lumière des différentes formes de spectatorat qui sont engendrées par les manifestations spatiales et sociales de la culture visuelle dans le monde quotidien. Le concept de «cinéma récent" est modelé sur la notion de cinéma d'archives, dans lequel les films sont refaits électroniquement et visionnés à travers une diversité d'architectures. Cette expression pourrait bien se révéler meilleure que celle de "cinéma postmoderne", car elle suggère l'idée de transition plutôt que de conclusion, de cycles plutôt que de ruptures, et elle désigne ce curieux isomorphisme entre le cinéma et le $\mathrm{XX}^{\mathrm{e}}$ siècle comme fondement d'une vision alternative de la modernité.

\section{Traduit de l'anglais par François Primeau et Denis Simard}

Concordia University

\section{NOTE}

1 Une version plus ślaborée du présent article paraîtra en anglais, sous le titre: "Parallax Historiography: The Flâneuse as Cyberfeminist", dans Scope: An On-Line Journal of Film Studies [<http://www.nottingham.ac.uk/film/journal/>http:// www.nottingham.ac.uk/film/journal/ ].

\section{OUVRAGES CITÉS}

Baudelaire, Charles. The Painter of Modern Life and other Essays. New York: Garland Publishing, 1978.

Benjamin, Walter. "N [Theoritics of Knowledge; Theory of Progress] ", Philosophy, Aesthetics, History. Chicago : University of Chicago Press, 1983a.

Benjamin, Walter, Charles Baudelaire: A Lyrical Poet in the Era of High Capitalism. Londres: Verso, $1983 \mathrm{~b}$. 
Benjamin, Walter. Illuminations (1935). New York: Schocken Books, 1969.

Bruno, Giuliana. "Site-Seeing: Architecture and the Moving Image". Wide Angle, vol. 19, n० 4, (1997), p. 8-24.

Bruno, Giuliana. Streetwalking on a Ruined Map: Cultural Theory and the City Films of Elvira Notari. Princeton: Princeton University Press, 1993.

Corrigan, Timothy. "Immediate History: Video Tape Interventions and Narrative Film ", dans Dudley Andrew (direction), The Image in Dispute: Art and Cinema in the Age of Photography. Austin: University of Texas Press (1997), p. 302-327.

Doane, Mary Ann. "Temporality, Storage, Legibility: Freud, Marey, and the Cinema ". Critical Inquiry, n 22 (1996), p. 313-343.

Doane, Mary Ann. "Information, Crisis, Catastrophe», dans Patricia Mellencamp (direction), Logics of Television: Essays in Cultural Criticism. Bloomington: Indiana University Press (1990), p. 222-239.

Friedberg, Anne, Window Shopping: Cinema and the Postmodern. Berkeley: University of California Press, 1993.

Gelber, Anke. "Women on the Screens and Streets of Modernity: In Search of the Female Flâneur", dans Dudley Andrew (direction), The Image in Dispute: Art and Cinema in the Age of Photography. Austin: University of Texas Press (1997), p. 55-86.

Gunning, Tom. "The Whole Town's Gawking: Early Cinema and the Visual Experience of Modernity". The Yale Journal of Criticism, vol. 7, $\mathrm{n}^{\circ} 2$ (1994), p. 189-204.

Gunning, Tom. "An Aesthetic of Astonishment: Film and the (In)Credulous Spectator ". Art \& Text, n 34 (1989), p 31-45.

Gunning, Tom. "The Cinema of Attractions: Early Film, its Spectator and the Avant-Garde", dans Thomas Elsaesser (direction), Early Cinema, Space, Frame, Narrative. Londres: British Film Institute (1991), p. 56-62.

Hansen, Miriam. "Early Cinema, Late Cinema: Transformations of the Public Sphere ", dans Linda Williams (direction), Viewing Positions: Ways of Seeing Film. New Brunswick: Rutgers University Press (1994), p. 134-152.

Hansen, Miriam. Babel \& Babylon: Spectatorship in American Silent Film. Cambridge: Harvard University Press, 1991.

Mulvey, Laura. "Visual Pleasure and Narrative Cinema". Screen, vol. 16, n 3 (1975), p. 6-18.

Rony, Fatimah Tobing. The Third Eye: Race, Cinema and Ethnographic Spectacle. Durham: Duke University Press, 1996.

Singer, Ben. "Modernity, Hyperstimulation, and the Rise of Popular Sensationalism ", dans Leo Charney et Vanessa R. Schwartz (direction), Cinema and the Invention of Modern Life. Berkeley: University of California Press (1995), p. 72-99.

Williams, Linda (direction). Viewing Positions: Ways of Seeing Film. New Brunswick: Rutgers University Press, 1994.

Wolff, Janet. Feminine Sentences: Essays on Women and Culture. Berkeley: University of California Press, 1990.

\section{SITES INTERNET}

American Mutoscope and Bioscope Co. http://www.muto 1895.com/lobby.html (2 mars 1999).

Baudrillard, Jean. "Reversion of History". Ctheory, http://www.ctheory.com/areversion_of_history.html (2 fév. 1999). 